\title{
Tetraquark and Pentaquark Systems in Lattice QCD
}

\author{
Fumiko Okiharu1, Takumi Doi ${ }^{2}$, Hiroko Ichie ${ }^{3}$, Hideaki Iida ${ }^{4}$, Noriyoshi Ishii ${ }^{5}$, \\ Makoto Oka ${ }^{3}$, Hideo Suganuma ${ }^{6}$, Toru T. Takahashi ${ }^{7}$ \\ ${ }^{1}$ Faculty of Education, Niigata University, Niigata, Japan \\ ${ }^{2}$ Theoretical Research Division, Nishina Center, RIKEN, Wako, Japan \\ ${ }^{3}$ Department of Physics, Tokyo Institute of Technology, Tokyo, Japan \\ ${ }^{4}$ Research and Education Center for Natural Sciences, Keio University, Kanagawa, Japan \\ ${ }^{5}$ Research Center for Nuclear Physics (RCNP), Osaka University, Ibaraki, Japan \\ ${ }^{6}$ Department of Physics, Graduate School of Science, Kyoto University, Kyoto, Japan \\ ${ }^{7}$ Gunma National College of Technology, Maebashi, Japan \\ Email: suganuma@scphys.kyoto-u.ac.jp
}

Received 12 February 2016; accepted 26 April 2016; published 29 April 2016

Copyright @ 2016 by authors and Scientific Research Publishing Inc.

This work is licensed under the Creative Commons Attribution International License (CC BY).

http://creativecommons.org/licenses/by/4.0/

(c) (i) Open Access

\begin{abstract}
We study multi-quark systems in lattice QCD. First, we revisit and summarize our accurate mass measurements of low-lying 5Q states with $J=1 / 2$ and $I=0$ in both positive- and negative-parity channels in anisotropic lattice QCD. The lowest positive-parity $5 Q$ state is found to have a large mass of about $2.24 \mathrm{GeV}$ after the chiral extrapolation. To single out the compact $5 \mathrm{Q}$ state from $\mathrm{NK}$ scattering states, we use the hybrid boundary condition (HBC), and find no evidence of the compact $5 \mathrm{Q}$ state below $1.75 \mathrm{GeV}$ in the negative-parity channel. Second, we study the multi-quark potential in lattice $Q C D$ to clarify the inter-quark interaction in multi-quark systems. The $5 Q$ potential $V_{5 Q}$ for the $Q Q-\bar{Q}-Q Q$ system is found to be well described by the "OGE Coulomb plus multi-Y Ansatz": The sum of the one-gluon-exchange (OGE) Coulomb term and the multi-Y-type linear term based on the flux-tube picture. The $4 Q$ potential $V_{4 Q}$ for the $Q Q-\bar{Q} \bar{Q}$ system is also described by the OGE Coulomb plus multi-Y Ansatz, when $Q Q$ and $\bar{Q} \bar{Q}$ are well separated. The $4 Q$ system is described as a "two-meson" state with disconnected flux tubes, when the nearest quark and antiquark pair are spatially close. We observe a lattice-QCD evidence for the "flip-flop", i.e., the fluxtube recombination between the connected $4 Q$ state and the "two-meson" state. On the confinement mechanism, the lattice QCD results indicate the flux-tube-type linear confinement in multi-quark hadrons. Finally, we propose a proper quark-model Hamiltonian based on the lattice QCD results.
\end{abstract}

\section{Keywords}

Lattice QCD, Multi-Quarks, Quark Confinement, Exotic Hadrons

How to cite this paper: Okiharu, F., Doi, T., Ichie, H., lida, H., Ishii, N., Oka, M., Suganuma, H. and Takahashi, T.T. (2016) Tetraquark and Pentaquark Systems in Lattice QCD. Journal of Modern Physics, 7, 774-789.

http://dx.doi.org/10.4236/jmp.2016.78072 


\section{Introduction}

The Multi-quark physics is one of the new interesting fields in the hadron physics. So far, several new particles have been experimentally reported as the candidates of multi-quark hadrons.

At first, the candidates of pentaquark (5Q) baryons were reported: a narrow peak identified as the $\Theta^{+}(1540)$ was found at SPring-8 [1], ITEP, JLab and ELSA [2]-[4]. The $\Theta^{+}(1540)$ has the baryon number $B=1$ and the strangeness $S=+1$, and hence it is a manifestly exotic baryon and is considered to be a pentaquark ( uudd $\bar{s}$ ) in the valence-quark picture. Other pentaquark candidate, the $\Xi^{--}$(1862) (ddssū ), was reported at CERN [5], and also a charmed pentaquark, the $\Theta_{c}(3099)$ ( uudd $\bar{c}$ ), was reported at HERA [6]. However, after highenergy experimental groups reported no evidence of the $\Theta^{+}(1540)$ [7]-[9], these pentaquark candidates are no more credible experimentally at present. (For the recent experimental status of the $\Theta^{+}(1540)$, see, e.g., Refs. [10] [11]). Nevertheless, the very $\Theta^{+}(1540)$ gave an important trigger to open the new area of the multiquark physics.

As the next important stage, the candidates of tetraquark (4Q) mesons were experimentally observed. The $\mathrm{X}(3872)$ [12]-[15] was found in the process of $\mathrm{B}^{+} \rightarrow \mathrm{K}^{+}+\mathrm{X}(3872) \rightarrow \mathrm{K}^{+}+\pi^{-} \pi^{+} J / \psi$ at KEK [12]. The $\mathrm{X}(3872)$ is much heavier than the $J / \psi$, and its mass is close to the threshold of $\mathrm{D}^{0}(\mathrm{cu})$ and $\overline{\mathrm{D}}^{0^{*}}(\mathrm{u} \overline{\mathrm{c}})$. However, its decay width is very narrow as $\Gamma<2.3 \mathrm{MeV}$ (90\% C.L.). These features indicate the $\mathrm{X}(3872)$ to be a tetraquark, e.g., a bound state of $\mathrm{D}^{0}$ and $\overline{\mathrm{D}}^{0 *}$. Similarly, the $\mathrm{D}_{s}(2317)$ [16] [17] is expected to be a tetraquark candidate. Also, quite recently, the LHCb experimental group has reported two candidates of the charmed pantaquark ( uudc $\bar{c}), \mathrm{P}_{c}^{+}(4380)$ and $\mathrm{P}_{\mathrm{c}}^{+}(4450)$ [18], from a careful analysis of the decay product in the high-energy process, and this news has activated the multi-quark physics again. In any case, these discoveries of multi-quark hadrons are expected to reveal hidden aspects of hadron physics.

In the theoretical side, the quark model is one of the most popular models to describe hadrons. In the quark model, mesons and baryons are usually described as $q \bar{q}$ and $3 q$ composite particles, respectively. In more microscopic viewpoint, quantum chromodynamics (QCD) is the fundamental theory to describe the strong interaction. In terms of QCD, not only ordinary $q \bar{q}$ mesons and $3 q$ baryons, but also exotic hadrons, such as multi-quark hadrons ( $q \bar{q} q \bar{q}, q q q q \bar{q}, \cdots)$, hybrid mesons $(q \bar{q} g, \cdots)$, hybrid baryons ( $q q q g, \cdots)$ and glueballs $(g g, g g g, \cdots)$ are expected to appear. We here aim to study these multi-quark hadrons directly based on QCD. Even at present, however, it is rather difficult to deal with the low-energy region analytically in QCD owing to its strong-coupling nature. As an alternative way, the lattice QCD Monte Carlo simulation is established as the powerful method to treat non-perturbative nature of low-lying hadrons including exotic hadrons. In this paper, we perform the following two lattice QCD studies to clarify the properties of multi-quark systems.

First, we investigate the mass and the parity of the 5Q system in lattice QCD. As for the parity assignment of the lowest-lying pentaquark, little agreement is achieved even in the theoretical side: the positive-parity assignment is supported by the chiral soliton model [19] and the diquark model [20], while the negative-parity assignment is supported by the nonrelativistic quark model [21], the QCD sum rule [22] and so on. For the exotic hadrons, most investigations have been done with model calculations, but these models were originally constructed only for ordinary hadrons. In fact, it is nontrivial that these models can describe the multi-quark system beyond the ordinary hadrons. To get solid information for the multi-quark systems, we study their properties directly from QCD by the lattice QCD simulation [23] [24], which is the first-principle calculation and model independent.

Second, we study the inter-quark interaction in multi-quark systems in lattice QCD. The inter-quark force is one of the most important elementary quantities in hadron physics. Nevertheless, for instance, no body knows the exact form of the confinement force in the multi-quark systems directly from QCD. In fact, some hypothetical forms of the inter-quark potential have been used in almost all quark model calculations so far. Then, the lattice QCD study of the inter-quark interaction is quite desired for the study of the multi-quark systems. It presents the proper Hamiltonian in multi-quark systems and leads to a guideline to construct the QCD-based quark model. In this paper, to clarify the inter-quark force in the multi-quark system, we study the 
static multi-quark potential systematically in lattice QCD using the multi-quark Wilson loop. We investigate the three-quark (3Q) potential [25]-[28], which is responsible to baryon properties, and perform the lattice-QCD study for the multi-quark potential, the tetraquark (4Q) and the pentaquark (5Q) potentials [29]-[33].

We show in Figure 1 our global strategy to understand the hadron properties from QCD. One way is the direct lattice QCD calculations for the low-lying hadron masses and simple hadron matrix elements, although the wave function is unknown and the practically calculable quantities are severely limited. The other way is to construct the quark model from QCD. From the analysis of the inter-quark forces in lattice QCD, we extract the quark-model Hamiltonian. Through the quark model calculation, one can obtain the quark wave-function of hadrons and more complicated properties of hadrons including properties of excited hadrons.

This paper is organized as follows. In Section 2, we present an accurate mass calculation of low-lying $5 \mathrm{Q}$ systems in anisotropic lattice QCD [34] [34]. In Section 3, we perform the systematic study of the inter-quark interaction in multi-quark systems [28]-[33]. Section 4 is devoted for the summary and concluding remarks.

\section{Lattice QCD Study for Multi-Quark Hadrons}

There have been many theoretical studies for multi-quark systems in the context of X(3872) and $\Theta^{+}(1540)$ [36] [37]. As for the $\Theta^{+}(1540)$, however, its existence as a low-lying pentaquark resonance is not credible experimentally. In fact, high-energy experimental groups reported no evidence of the $\Theta^{+}(1540)$ [7]-[9].

Also in lattice QCD, there is no consensus on the existence and the parity assignment of the lowest-lying pentaquark system. Two early works supported the negative-parity state for the $\Theta^{+}(1540)$ [38] [39], while one early work supported the positive-parity state [39]. We and another group indicated no evidence for the low-lying pentaquark narrow resonance [34] [41], and one study suggested a negative-parity pentaquark state in more highly-excited region around $1.8 \mathrm{GeV}$ [42].

In this section, we perform the accurate mass measurement of the $5 \mathrm{Q}$ system in anisotropic lattice QCD, and apply hybrid boundary condition [34] [43] to distinguish a compact resonance and a scattering state.

\subsection{Strategy for High Precession Measurements in Lattice QCD}

As a difficulty on the lattice study of multi-quarks, even if a compact multi-quark resonance state exists, there appears a mixture with several multi-hadron scattering states, even at the quenched level. For instance, in the channel of $\Theta^{+}$, several NK scattering states appears. In this paper, we use the term of the $\Theta^{+}$only for the compact $5 \mathrm{Q}$ resonance to distinguish it from the NK scattering state. In order to examine whether the low-lying $5 \mathrm{Q}$ state appears as a compact resonance $\Theta^{+}$, we perform the accurate lattice QCD calculations with adopting the following three advanced methods [34].

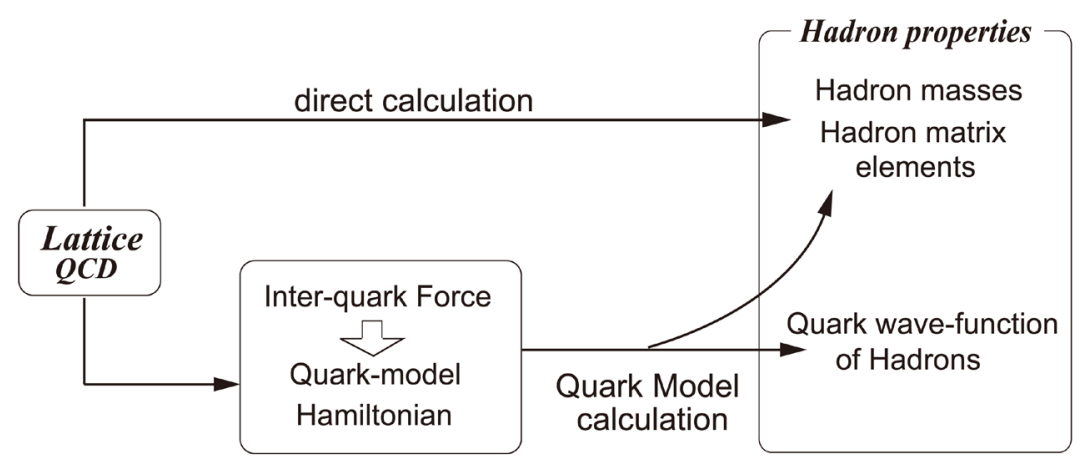

Figure 1. Our global strategy to understand the hadron properties from QCD. One way is the direct lattice QCD calculations for the low-lying hadron masses and simple hadron matrix elements, although the wave function is unknown and the practically calculable quantities are severely limited. The other way is to construct the quark model from QCD. From the analysis of the inter-quark forces in lattice QCD, we extract the quark-model Hamiltonian. Through the quark model calculation, one can obtain the quark wave-function of hadrons and more complicated properties of hadrons including properties of excited hadrons. 


\subsubsection{Usage of Anisotropic Lattice QCD}

We use the anisotropic lattice, where the temporal lattice spacing $a_{t}$ is much finer than the spatial one $a_{s}$ as shown in Figure 2. In lattice QCD, hadron masses are calculated from the asymptotic temporal behavior of the hadron correlators. On the anisotropic lattice, we can get the detailed information on the temporal behavior of the 5Q correlator, and hence we can perform accurate mass measurements for the low-lying 5Q system.

\subsubsection{Usage of the Non-NK-type Interpolating Field Operator}

We use a non-NK-type interpolating field to extract the $\Theta^{+}(1540)$ state. This choice of the interpolating field would be important and effective. For instance, in Ref. [41], the authors used the NK-type interpolating field and only obtained the NK scattering state instead of the compact 5Q state. However, their null result may be merely due to a small amount of the compact 5Q component in the NK-type interpolating field, because their calculation suffers from a large contamination of NK scattering states.

We adopt the non-NK-type interpolating field [22],

$$
O_{\alpha} \equiv \epsilon_{a b c} \epsilon_{a d e} \epsilon_{b f g}\left(u_{d}^{T} C \gamma_{5} d_{e}\right)\left(u_{f}^{T} C d_{g}\right)\left(C \bar{s}_{c}^{T}\right)_{\alpha},
$$

for the 5Q state with spin $J=1 / 2$ and isospin $I=0$. Here $\alpha$ denotes the Dirac index, and roman indices $a-g$ are color indices. $C \equiv \gamma_{4} \gamma_{2}$ denotes the charge conjugation matrix. Note that the non-NK-type operator in Equation (1) cannot be decomposed into $\mathrm{N}$ and $\mathrm{K}$ in the nonrelativistic limit and its coupling to the NK state is rather weak. Hence, the $5 \mathrm{Q}$ resonance state $\Theta^{+}$can be singled out as much as possible in the present calculation, and the results are less biased by the contamination from NK scattering states.

\subsubsection{Application of the Hybrid Boundary Condition Method}

To distinguish compact resonances from scattering states, we have proposed a useful method with the "hybrid boundary condition" (HBC) [34] [43] instead of the ordinary periodic boundary condition. In the HBC, we impose the it anti-periodic boundary condition for $\mathrm{u}, \mathrm{d}$ quarks, and the periodic boundary condition for s-quarks, as shown in Table 1. By applying the HBC on a finite-volume lattice, the NK threshold is raised up, while the mass of a compact 5Q resonance $\Theta^{+}$is almost unchanged. Therefore, we can distinguish a compact 5Q state $\Theta^{+}$from an NK scattering state by comparing between the HBC and the standard periodic boundary condition.

In lattice QCD with the finite spatial volume $L^{3}$, the spatial momenta are quantized as $p_{i}=2 n_{i} \pi / L$ $\left(n_{i} \in \mathbf{Z}\right.$ ) under the periodic boundary condition and $p_{i}=\left(2 n_{i}+1\right) \pi / L$ under the anti-periodic boundary condition. In the periodic boundary condition, $\mathrm{N}$ and $\mathrm{K}$ can have zero momenta $\left|\mathbf{p}_{\min }\right|=0$ in the s-wave NK scattering state. The HBC imposes the anti-periodic boundary condition for $\mathrm{u}$ and $\mathrm{d}$ quarks and periodic boundary condition for s quark, while the periodic boundary condition is usually employed for all $\mathrm{u}, \mathrm{d}$, s quarks. In the HBC, the net boundary conditions of both $\mathrm{N}$ (uud,udd) and $\mathrm{K}(\mathrm{u} \overline{\mathrm{s}}, \mathrm{d} \overline{\mathrm{s}})$ are anti-periodic. Then, under the HBC, $N$ and $K$ have minimum momenta $p_{i}=\sqrt{3} \pi / L$ in a finite box with $L^{3}$, and the threshold of the s-wave NK scattering state is raised up as $\sqrt{m_{\mathrm{N}}^{2}+\mathbf{p}_{\min }^{2}}+\sqrt{m_{\mathrm{K}}^{2}+\mathbf{p}_{\min }^{2}}$. In contrast to $\mathrm{N}$ and $\mathrm{K}$, the compact $5 \mathrm{Q}$ resonance $\Theta^{+}$( uudd $\bar{s}$ ) contains even number of $u$ and d quarks, and hence its mass does not shift in the HBC (see Table 2).
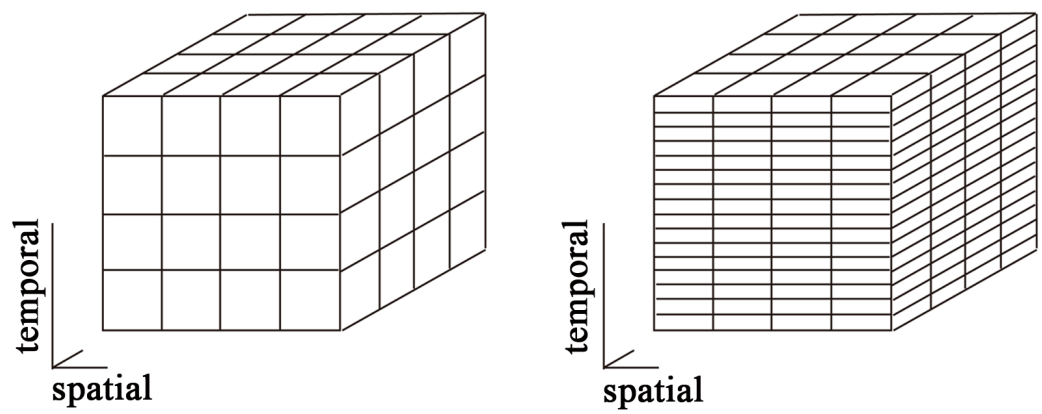

Figure 2. Schematic figures of the isotropic lattice (left) and the anisotropic lattice (right). On the anisotropic lattice, the temporal lattice spacing $a_{t}$ is taken to be smaller than the spatial one $a_{s}$. 
Table 1. The hybrid boundary condition (HBC) to distinguish a compact multi-quark resonance and an two-hadron scattering state for the uudd $\bar{s}$ system. The standard boundary condition (BC) is also shown for comparison.

\begin{tabular}{cccc}
\hline & u quark & d quark & s quark \\
\hline HBC & anti-periodic & anti-periodic & periodic \\
standard BC & periodic & periodic & periodic \\
\hline
\end{tabular}

Table 2. The net boundary condition for $\Theta^{+}$( uudd $\bar{s}$ ), $N$ (uud or udd) and $K(d \bar{s}$ or $u \bar{s}$ ) in the hybrid boundary condition (HBC) and in the standard boundary condition (BC).

\begin{tabular}{cccc}
\hline & $\Theta^{+}($uudd $\bar{s})$ & $\mathrm{N}$ (uud or udd) & $\mathrm{K}(\mathrm{d} \overline{\mathrm{s}}$ or $\mathrm{us})$ \\
\hline HBC & periodic & anti-periodic & anti-periodic \\
standard BC & periodic & periodic & periodic \\
\hline
\end{tabular}

\subsection{Lattice QCD Setup for the Pentaquark Mass}

To generate gluon configurations, we use the standard plaquette action on the anisotropic lattice as [34]

$$
S_{\mathrm{G}}=\frac{\beta}{N_{c}} \frac{1}{\gamma_{\mathrm{G}}} \sum_{s, i<j \leq 3} \operatorname{Re} \operatorname{Tr}\left\{1-P_{i j}(s)\right\}+\frac{\beta}{N_{c}} \gamma_{\mathrm{G}} \sum_{s, i \leq 3} \operatorname{Re} \operatorname{Tr}\left\{1-P_{i 4}(s)\right\},
$$

with $\beta \equiv 2 N_{c} / g^{2}$, the plaquette $P_{\mu v}(s)$ and the bare anisotropy $\gamma_{\mathrm{G}}$.

For the quark part, we adopt the $O(a)$-improved Wilson (clover) fermion action on the anisotropic lattice,

$$
S_{\mathrm{F}} \equiv \sum_{x, y} \bar{\psi}(x) K(x, y) \psi(y),
$$

with the quark kernel $K(x, y)$ as

$$
\begin{aligned}
K(x, y) \equiv & \delta_{x, y}-\kappa_{t}\left\{\left(1-\gamma_{4}\right) U_{4}(x) \delta_{x+\hat{4}, y}+\left(1+\gamma_{4}\right) U_{4}^{\dagger}(x-\hat{4}) \delta_{x-\hat{4}, y}\right\} \\
& -\kappa_{s} \sum_{i}\left\{\left(r-\gamma_{i}\right) U_{i}(x) \delta_{x+\hat{i}, y}+\left(r+\gamma_{i}\right) U_{i}^{\dagger}(x-\hat{i}) \delta_{x-\hat{i}, y}\right\} \\
& -\kappa_{s} c_{E} \sum_{i} \sigma_{i 4} G_{i 4} \delta_{x, y}-r \kappa_{s} c_{B} \sum_{i<j} \sigma_{i j} G_{i j} \delta_{x, y},
\end{aligned}
$$

where $\kappa_{s}$ and $\kappa_{t}$ denote the spatial and temporal hopping parameters, respectively. $G_{\mu v}$ denotes the field strength, which is defined through the standard clover-leaf-type construction. The Wilson parameter $r$ and the clover coefficients, $c_{E}$ and $c_{B}$, are fixed by the tadpole-improved tree-level values as $r=1 / \xi, c_{E}=1 /\left(u_{s} u_{t}^{2}\right)$ and $C_{B}=1 / u_{s}^{3}$, where $u_{s}$ and $u_{t}$ denote the mean-field values of the spatial and the temporal link-variables, respectively.

For the lattice QCD simulation, we use $\beta=5.75$ and $12^{3} \times 96$ with the renormalized anisotropy $a_{s} / a_{t}=4$, which corresponds to $\gamma_{\mathrm{G}}=3.2552$. In this calculation, the lattice spacing is found to be $a_{s} \simeq 0.18 \mathrm{fm} \simeq(1.1 \mathrm{GeV})^{-1}$ and $a_{t} \simeq 0.045 \mathrm{fm} \simeq(4.4 \mathrm{GeV})^{-1}$. We adopt four values of the hopping parameter as $\kappa=0.1210(0.0010) 0.1240$ for $\mathrm{u}$ and d quarks, and use $\kappa_{\text {s-quark }}=0.1240$ for the s quark. We calculate typical hadron masses at each $\kappa$ as shown in Table 3, and find $\kappa_{\text {phys. }} \simeq 0.1261$ corresponding to the physical situation of $m_{\pi} \simeq 0.14 \mathrm{GeV}$.

\subsection{Lattice QCD Results for the $\Theta^{+}(1540)$}

Now, using anisotropic lattice QCD, we perform the accurate mass measurement of the low-lying 5Q states with 
Table 3. The lattice QCD results for the masses of $\pi, \rho, \mathrm{K}$ and $\mathrm{N}$ at each hopping parameter $\kappa$ in the physical unit of $\mathrm{GeV}$ [43]. The $\kappa_{\text {phys. }} \simeq 0.1261$ corresponds to the physical situation of $m_{\pi} \simeq 0.14 \mathrm{GeV}$.

\begin{tabular}{cccccc}
\hline$\kappa$ & 0.1210 & 0.1220 & 0.1230 & 0.1240 & $\kappa_{\text {phys. }}$ \\
\hline$m_{\pi}$ & $1.005(2)$ & $0.898(2)$ & $0.784(2)$ & $0.656(3)$ & 0.140 \\
$m_{\rho}$ & $1.240(3)$ & $1.161(3)$ & $1.085(4)$ & $1.011(5)$ & $0.850(7)$ \\
$m_{K}$ & $0.845(2)$ & $0.785(2)$ & $0.723(2)$ & $0.656(3)$ & $0.530(4)$ \\
$m_{N}$ & $1.878(5)$ & $1.744(5)$ & $1.604(5)$ & $1.460(6)$ & $1.173(9)$ \\
\hline
\end{tabular}

$J=1 / 2$ and $I=0$ in both positive- and negative-parity channels from the correlator of the non-NK-type 5Q operator with parity projection [34].

In Figure 3, we show the lattice QCD results [34] for the masses of lowest positive- and negative-parity 5Q states under the standard periodic boundary condition. After the chiral extrapolation, the lowest positive-parity $5 \mathrm{Q}$ state is found to be rather heavy as $m_{5 \mathrm{Q}}\left(J^{\pi}=\frac{1^{+}}{2}\right) \simeq 2.24 \mathrm{GeV}$, which seems to be too heavy to be identified as the $\Theta^{+}(1540)$.

On the other hand, we get a lower mass for the negative-parity $5 \mathrm{Q}$ state as $m_{5 \mathrm{Q}}\left(J^{\pi}=\frac{1^{-}}{2}\right) \simeq 1.75 \mathrm{GeV}$ after the chiral extrapolation. This value $m_{5 \mathrm{Q}} \simeq 1.75 \mathrm{GeV}$ seems to be closer to the experimental result of $m_{\Theta^{+}} \simeq 1.54 \mathrm{GeV}$. At this stage, however, this lowest negative-parity 5Q state may be merely an NK scattering state, although the non-NK-type $5 \mathrm{Q}$ operator used in this calculation includes only a small amount of the NK component.

To clarify whether the observed low-lying $5 \mathrm{Q}$ state is a compact $5 \mathrm{Q}$ resonance $\Theta^{+}$or an NK scattering state, we use the hybrid boundary condition (HBC) method, and compare the lattice results with the HBC and those with the standard periodic boundary condition (BC). Recall that, in the HBC, the NK threshold is largely raised up, while the mass of the compact $5 \mathrm{Q}$ resonance (uudd $\overline{\mathrm{s}}$ ) is to be almost unchanged, as was mentioned in Section 2.1.3.

In Figure 4, we show the mass of the lowest-lying negative-parity 5Q state in lattice QCD with the standard periodic BC and the HBC at each $\kappa$ [34]. The symbols denote the lattice QCD results for the 5Q state and the lines denote the NK threshold at each $\kappa$. The left and right figures show the results with the standard periodic BC and the hybrid boundary condition (HBC), respectively. Note that the NK threshold is estimated to be raised up about $200-250 \mathrm{MeV}$ in the HBC.

As a lattice QCD result, the mass of the $5 \mathrm{Q}$ state is largely raised in the HBC in accordance with the NK threshold, which indicates that the lowest $5 \mathrm{Q}$ state observed on the lattice is merely an s-wave NK scattering state. In other words, if there exists a compact $5 \mathrm{Q}$ resonance $\Theta^{+}$below $1.75 \mathrm{GeV}$, it should be observed in this lattice calculation with the non-NK-type operator, and its mass should be almost unchanged also in the HBC. However, there is no such a $5 \mathrm{Q}$ state observed in the lattice calculation, which means absence of the compact $5 \mathrm{Q}$ resonance $\Theta^{+}$below $1.75 \mathrm{GeV}$.

To conclude, our lattice QCD calculation at the quenched level indicates absence of the low-lying compact $5 \mathrm{Q}$ resonance $\Theta^{+}$with $J=1 / 2$ and $I=0$ near $1.54 \mathrm{GeV}$ [34].

\subsection{Discussion on Null Result of $\Theta^{+}(1540)$ in Lattice QCD}

Now, let us consider the physical consequence of the present null result on the low-lying $5 \mathrm{Q}$ resonance $\Theta^{+}$in lattice QCD. One plausible answer is absence of the pentaquark resonance $\Theta^{+}(1540)$, as was indicated by several experiments [7]-[11]. However, there may be some loopholes in the lattice calculation.

First, the present lattice simulation has been done at the quenched level, where dynamical quark effects are suppressed. This quenching effect is not clear and then it may cause the 5Q resonance $\Theta^{+}$to be heavier as an unknown effect. 


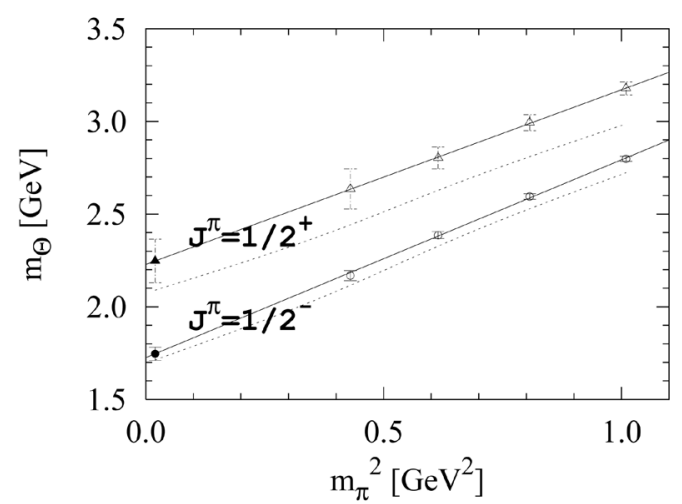

Figure 3. The lowest mass $m_{5 \mathrm{Q}}$ of the positive- and negative-parity 5Q states plotted against $m_{\pi}^{2}$, taken from Ref. [34]. The open symbols denote the direct lattice QCD data for positive-parity (triangles) and negative-parity (circles). The solid symbols denote the results of the chiral extrapolation. The dotted lines indicate the NK thresholds for p-wave (upper) and s-wave (lower) cases.

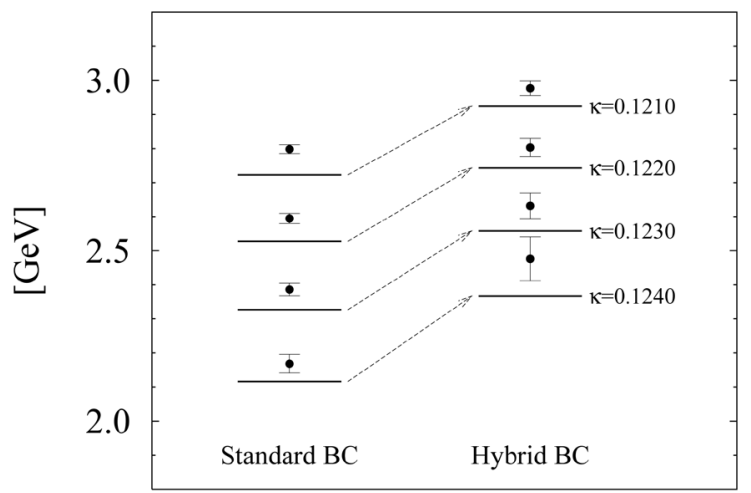

Figure 4. Comparison between the standard periodic boundary condition (Standard BC) and the Hybrid Boundary Condition (HBC) for the lowest mass of the negative-parity 5Q system, taken from Ref. [34]. At each $\kappa$, the lattice QCD result (the solid symbol) is raised up in accordance with the corresponding NK threshold (the solid line). This behavior indicates that the low-lying negative-parity $5 \mathrm{Q}$ state observed in lattice QCD is an NK scattering state rather than a compact $5 \mathrm{Q}$ resonance $\Theta^{+}$.

Second, we investigated the $5 \mathrm{Q}$ state with spin $J=1 / 2$ and isoscalar $I=0$ in this paper. However, the $\Theta^{+}(1540)$ may have other quantum numbers [44]-[46], e.g., spin $J=3 / 2$, isovector $I=1$ or isotensor $I=2$. Considering such a possibility, our group investigated the $5 Q$ system with higher spin $J=3 / 2$ in lattice QCD, and found no low-lying pentaquark also in this channel [43].

Third, we have used a localized 5Q interpolating field in this lattice QCD calculation. However, the actual $\Theta^{+}(1540)$ may have more complicated structure beyond the localized interpolating field. Such a possibility has been pointed out in the theoretical side. For instance, Karliner and Lipkin [47] proposed the diquark-triquark ( $q q-q q \bar{q}$ ) picture for the $\Theta$, and Bicudo et al. [48] pointed out the possibility of the heptaquark picture, where the $\Theta^{+}$is described as a bound state of $\pi, \mathrm{K}$ and $\mathrm{N}$. If the $\Theta^{+}(1540)$ has such a complicated structure, we have to use the corresponding nonlocal interpolating field to get its proper information.

\subsection{Necessity of the Wave Function of Multi-Quarks}

So far, we have performed the direct mass measurement of $5 \mathrm{Q}$ states in lattice QCD, where the path integral over arbitrary states is numerically calculated on a supercomputer. In the path-integral formalism, however, it is rather difficult to extract the state information, such as the wave-function of the multi-quark state, and therefore 
only limited simple information can be obtained in the direct lattice-QCD calculation.

Actually, to distinguish the compact $5 \mathrm{Q}$ resonance $\Theta^{+}$from NK scattering states was rather difficult in lattice QCD, and hence we had to develop a new method with the hybrid boundary condition (HBC). In this respect, if the wave function is obtained, one easily finds out whether it is a compact resonance state $\Theta^{+}$or not.

Indeed, to get the wave function is very important to clarify the further various properties of the multi-quark state such as the underlying structure and the decay width, which cannot be obtained practically only with the direct lattice-QCD calculation.

Then, apart from the direct lattice-QCD calculation, we have to seek the way to obtain the proper wave function of the multi-quark state. To do so, we need a proper Hamiltonian for the multi-quark system based on QCD. One possible way in this direction is to construct the quark model from QCD, as was mentioned in Section 1. In the next section, we study the inter-quark interaction in multi-quark systems directly from QCD, and aim to construct the QCD-based quark-model Hamiltonian.

\section{Inter-Quark Interaction in Multi-Quark Systems in Lattice QCD}

In this section, we study the inter-quark interaction in multi-quark systems using lattice QCD [29]-[33], and seek for the QCD-based quark-model Hamiltonian to describe multi-quark hadrons. The quark-model Hamiltonian consists of the kinetic term and the potential term, which is not known form QCD in multi-quark systems.

As for the potential at short distances, the perturbative one-gluon-exchange (OGE) potential would be appropriate, due to the asymptotic nature of QCD. For the long-range part, however, there appears the confinement potential as a typical non-perturbative property of QCD, and its form is highly nontrivial in the multi-quark system.

In fact, to clarify the confinement force in multi-quark systems is one of the essential points for the construction of the QCD-based quark-model Hamiltonian. Then, in this paper, we investigate the multi-quark potential in lattice QCD, with paying attention to the confinement force in multi-quark hadrons.

\subsection{The Three-Quark Potential in Lattice QCD}

So far, only for the simplest case of static $Q \bar{Q}$ systems, detailed lattice QCD studies have been done, and the $\mathrm{QQ}$ potential $V_{\mathrm{Q} \overline{\mathrm{Q}}}$ is known to be well described by the Coulomb plus linear potential as [23]-[26] [49]

$$
V_{\mathrm{Q} \overline{\mathrm{Q}}}(r)=-\frac{A_{\mathrm{Q} \overline{\mathrm{Q}}}}{r}+\sigma_{\mathrm{Q} \overline{\mathrm{Q}}} r+C_{\mathrm{Q} \overline{\mathrm{Q}}}
$$

with $r$ being the inter-quark distance.

To begin with, we study three-quark (3Q) systems in lattice QCD to understand the structure of baryons at the quark-gluon level. Similar to the derivation of the $\mathrm{Q} \overline{\mathrm{Q}}$ potential from the Wilson loop, we calculate the $3 \mathrm{Q}$ potential $V_{3 Q}$ from the $3 \mathrm{Q}$ Wilson loop $W_{3 \mathrm{Q}}$ in SU(3) lattice QCD with $\left(\beta=5.7,12^{3} \times 24\right),(\beta=5.8$, $\left.16^{3} \times 32\right),\left(\beta=6.0,16^{3} \times 32\right)$ and $\left(\beta=6.2,24^{4}\right)$ at the quenched level. For more than 300 different patterns of spatially-fixed 3Q systems, we perform accurate and detailed calculations for the $3 Q$ potential [25]-[28] [31] [33], and find that the lattice QCD data of the $3 \mathrm{Q}$ potential $V_{3 \mathrm{Q}}$ are well described by the Coulomb plus Y-type linear potential, i.e., Y-Ansatz,

$$
V_{3 \mathrm{Q}}=-A_{3 \mathrm{Q}} \sum_{i<j} \frac{1}{\left|\mathbf{r}_{i}-\mathbf{r}_{j}\right|}+\sigma_{3 \mathrm{Q}} L_{\min }^{3 \mathrm{Q}}+C_{3 \mathrm{Q}},
$$

within 1\%-level deviation [25]-[28] [31] [33]. Here, $L_{\min }^{3 Q}$ is the minimal total length of the color flux tube, which is Y-shaped for the $3 \mathrm{Q}$ system.

To demonstrate the validity of the Y-Ansatz, we show in Figure 5 the lattice QCD data of the 3Q confinement potential $V_{3 Q}^{\text {conf }}$, i.e., $3 \mathrm{Q}$ potential subtracted by the Coulomb part, plotted against Y-shaped flux-tube length $L_{\min }^{3 Q}$ [30]. For each $\beta$, clear linear correspondence is found between 3Q confinement potential $V_{3 Q}^{\text {conf }}$ 


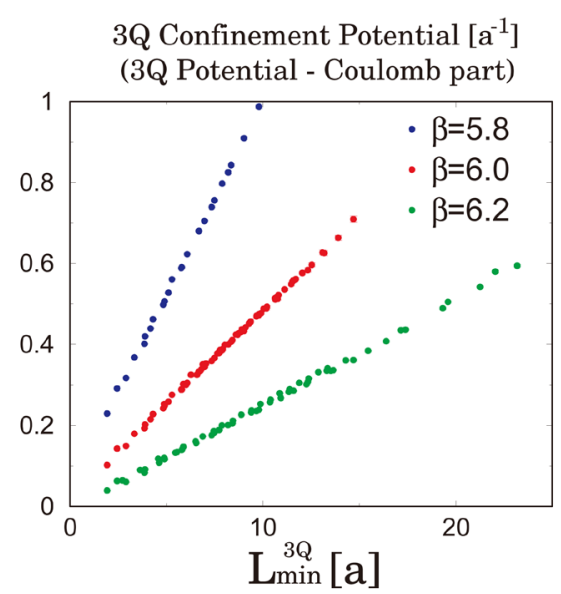

Figure 5. The lattice QCD result for the $3 \mathrm{Q}$ confinement potential $V_{3 \mathrm{Q}}^{\text {conf }}$, i.e., the $3 \mathrm{Q}$ potential subtracted by its Coulomb part, plotted against Y-shaped flux-tube length $L_{\min }^{3 Q}$ at $\beta=5.8,6.0$ and 6.2 in the lattice unit, taken from Ref. [30]. The clear linear correspondence between 3Q confinement potential $V_{3 \mathrm{Q}}^{\text {conf }}$ and $L_{\min }^{3 \mathrm{Q}}$ indicates the Y-Ansatz for the 3Q potential.

and $L_{\min }^{3 Q}$, which indicates the Y-Ansatz for the 3Q potential [30] [31] [33].

Here, we consider the physical meaning of the Y-Ansatz. Apart from an irrelevant constant, the Y-Ansatz, Equation (6), consists of the Coulomb term and the Y-type linear potential, which play the dominant role at short and long distances, respectively. The Coulomb term would originate from the one-gluon-exchange (OGE) process. In fact, at short distances, perturbative QCD is applicable, and therefore the inter-quark potential is expressed as the sum of the two-body one-gluon-exchange (OGE) Coulomb potential.

The appearance of the Y-type linear potential supports the flux-tube picture [50]-[53] at long distances, where there appears the color flux tube linking quarks inside hadrons with its length minimized. In particular, the confinement force in baryons corresponds to the Y-shaped flux tube, which implies existence of the three-body interaction in baryons.

In usual many-body systems, the main interaction is described by a two-body interaction and the three-body interaction is a higher-order contribution. In contrast, as is clarified by our lattice-QCD study, the quark confinement force in baryons is a genuinely three-body interaction [25] [26], which is one of significant features of QCD. In fact, the appearance of the Y-type junction and the three-body confinement force reflect the SU(3) group structure in QCD, e.g., the number of color, $N_{c}=3$, and is peculiar to QCD [25] [26]. In this sense, the study of the $3 \mathrm{Q}$ system is very important to get a deeper insight of the QCD physics.

In lattice QCD, a clear Y-type flux-tube formation is actually observed for spatially-fixed 3Q systems [31] [33] [54]. Thus, together with several other analytical and numerical studies [55]-[57], the Y-Ansatz seems to be confirmed as the correct functional form of the static $3 \mathrm{Q}$ potential. This result indicates the color-flux-tube picture for baryons.

\subsection{The OGE Coulomb Plus Multi-Y Ansatz}

Now, we proceed to multi-quark systems. We first consider the theoretical form of the multi-quark potential, since we will have to analyze the lattice QCD data by comparing them with some theoretical Ansatz.

By generalizing the lattice QCD result of the Y-Ansatz for the three-quark potential, we propose the one-gluon-exchange (OGE) Coulomb plus multi-Y Ansatz [29]-[33],

$$
V=\frac{g^{2}}{4 \pi} \sum_{i<j} \frac{T_{i}^{a} T_{j}^{a}}{\left|\mathbf{r}_{i}-\mathbf{r}_{j}\right|}+\sigma L_{\min }+C,
$$

for the potential form of the multi-quark system. Here, the confinement potential is proportional to the minimal total length $L_{\min }$ of the color flux tube linking the quarks, which is multi-Y shaped in most cases.

In the following, we study the inter-quark interaction in multi-quark systems in lattice QCD, and compare the 
lattice QCD data with the theoretical form in Equation (7). Note here that the lattice QCD data are meaningful as primary data on the multi-quark system directly based on QCD, and do not depend on any theoretical Ansatz.

\subsection{Formalism of the Multi-Quark Wilson Loop}

Next, we formulate the multi-quark Wilson loop to obtain the multi-quark potential in lattice QCD [29]-[33].

Similar to the derivation of the $\mathrm{Q} \overline{\mathrm{Q}}$ potential from the Wilson loop, the static multi-quark potential can be derived from the corresponding multi-quark Wilson loop. We construct the tetraquark Wilson loop $W_{4 \mathrm{Q}}$ [30] and the pentaquark Wilson loop $W_{5 \mathrm{Q}}$ [29] in a gauge invariant manner as shown in Figure 6(a) and Figure 6(b), respectively.

The tetraquark Wilson loop $W_{4 \mathrm{Q}}$ and the pentaquark Wilson loop $W_{5 \mathrm{Q}}$ are defined by

$$
\begin{aligned}
W_{4 \mathrm{Q}} & \equiv \frac{1}{3} \operatorname{tr}\left(\tilde{M}_{1} \tilde{R}_{12} \tilde{M}_{2} \tilde{L}_{12}\right), \\
W_{5 \mathrm{Q}} & \equiv \frac{1}{3 !} \epsilon^{a b c} \epsilon^{a^{\prime} b^{\prime} c^{\prime}} M^{a a^{\prime}}\left(\tilde{R}_{3} \tilde{R}_{12} \tilde{R}_{4}\right)^{b b^{\prime}}\left(\tilde{L}_{3} \tilde{L}_{12} \tilde{L}_{4}\right)^{c c^{\prime}},
\end{aligned}
$$

where $\tilde{M}, \quad \tilde{M}_{i}, \quad \tilde{L}_{j}$ and $\tilde{R}_{j}(i=1,2, j=1,2,3,4)$ are given by

$$
\tilde{M}, \tilde{M}_{i}, \tilde{R}_{j}, \tilde{L}_{j} \equiv P \exp \left\{i g \int_{M, M_{i}, R_{j}, L_{j}} \mathrm{~d} x^{\mu} A_{\mu}(x)\right\} \in \mathrm{SU}(3)_{\mathrm{c}} .
$$

Here, $\tilde{R}_{12}$ and $\tilde{L}_{12}$ are defined by

$$
\tilde{R}_{12}^{a^{\prime} a} \equiv \frac{1}{2} \epsilon^{a b c} \epsilon^{a^{\prime} b^{\prime} c^{\prime}} R_{1}^{b b^{\prime}} R_{2}^{c c^{\prime}}, \quad \tilde{L}_{12}^{a^{\prime} a} \equiv \frac{1}{2} \epsilon^{a b c} \epsilon^{a^{\prime} b^{\prime} c^{\prime}} L_{1}^{b b^{\prime}} L_{2}^{c c^{\prime}} .
$$

The multi-quark Wilson loop physically means that a gauge-invariant multi-quark state is generated at $t=0$ and annihilated at $t=T$ with quarks being spatially fixed in $\mathbf{R}^{3}$ for $0<t<T$.

The multi-quark potential is obtained from the vacuum expectation value of the multi-quark Wilson loop:

$$
V_{4 \mathrm{Q}}=-\lim _{T \rightarrow \infty} \frac{1}{T} \ln \left\langle W_{4 \mathrm{Q}}\right\rangle, \quad V_{5 \mathrm{Q}}=-\lim _{T \rightarrow \infty} \frac{1}{T} \ln \left\langle W_{5 \mathrm{Q}}\right\rangle .
$$

\subsection{Lattice QCD Setup for the Multi-Quark Potential}

Here, we briefly summarize the lattice QCD setup in this calculation. For the study of the multi-quark potential, the SU(3) lattice QCD simulation is done with the standard plaquette action at $\beta=6.0$ on the $16^{3} \times 32$ lattice at the quenched level. (The calculation for large-size multi-quark configurations are performed by identifying $16^{3} \times 32$ as the spatial size.)

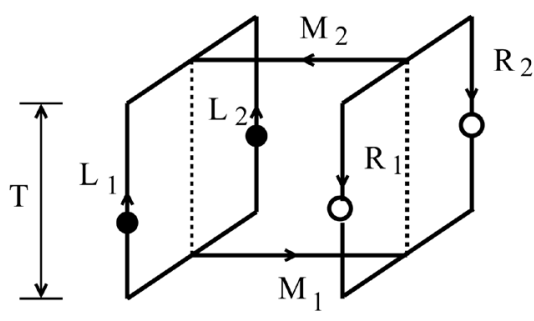

(a)

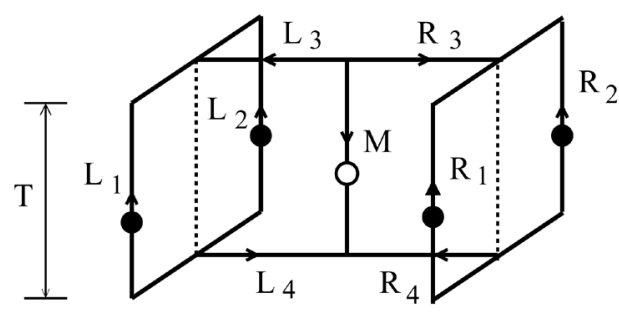

(b)

Figure 6. (a) The tetraquark Wilson loop $W_{4 Q}$ [30]. (b) The pentaquark Wilson loop $W_{5 \mathrm{Q}}$ [29]. The contours $M, M_{i}, R_{j}, L_{j}(i=1,2, j=3,4)$ are line-like and $R_{j}, L_{j}(j=1,2)$ are staple-like. The multi-quark Wilson loop physically means that a gauge-invariant multi-quark state is generated at $t=0$ and annihilated at $t=T$ with quarks being spatially fixed in $\mathbf{R}^{3}$ for $0<t<T$. 
In this calculation, the lattice spacing $a$ is estimated as $a \simeq 0.104 \mathrm{fm}$, which leads to the string tension $\sigma_{\mathrm{Q} \overline{\mathrm{Q}}}=(427 \mathrm{MeV})^{2}$ in the $\mathrm{Q} \overline{\mathrm{Q}}$ potential [31]. We use 150 gauge configurations for the $5 \mathrm{Q}$ potential simulation and 300 gauge configurations for the $4 \mathrm{Q}$ potential simulation. The smearing method is used for the enhancement of the ground-state component. We here adopt $\alpha=2.3$ and the iteration number $N_{\text {smr }}=40$, which lead to a large enhancement of the ground-state component [29]-[33].

\subsection{Lattice QCD Result of the Pentaquark Potential}

We study the pentaquark potential $V_{5 \mathrm{Q}}$ in lattice QCD for 56 different patterns of QQ- $\bar{Q}-\mathrm{QQ}$ type pentaquark configurations, as shown in Figure 7. As the conclusion, the lattice QCD data of $V_{5 \mathrm{Q}}$ are found to be well described by the OGE Coulomb plus multi-Y Ansatz, i.e., the sum of the OGE Coulomb term and the multiY-type linear term based on the flux-tube picture [29] [31]-[33].

We show in Figure 8 the lattice QCD results of the 5Q potential $V_{5 Q}$ [29] for symmetric planar 5Q configurations as shown in Figure 7, where each 5Q system is labeled by $d \equiv \overline{\mathrm{Q}_{1} \mathrm{Q}_{2}} / 2$ and $h \equiv \overline{\mathrm{Q}_{1} \mathrm{Q}_{3}}$.

In Figure 8, we add the theoretical curves of the OGE Coulomb plus multi-Y Ansatz, where the coefficients $\left(A_{5 \mathrm{Q}}, \sigma_{5 \mathrm{Q}}\right)$ are set to be $\left(A_{3 \mathrm{Q}}, \sigma_{3 \mathrm{Q}}\right)$ obtained from the 3Q potential [26]. (Note that there is no adjustable parameter in the theoretical Ansatz apart from an irrelevant constant.) In Figure 8, one finds a good agreement between the lattice QCD data of $V_{5 \mathrm{Q}}$ and the theoretical curves of the OGE Coulomb plus multi-Y Ansatz.

In this way, the pentaquark potential $V_{5 Q}$ is found to be well described by the OGE Coulomb plus multi-Y Ansatz as [29] [31]-[33].

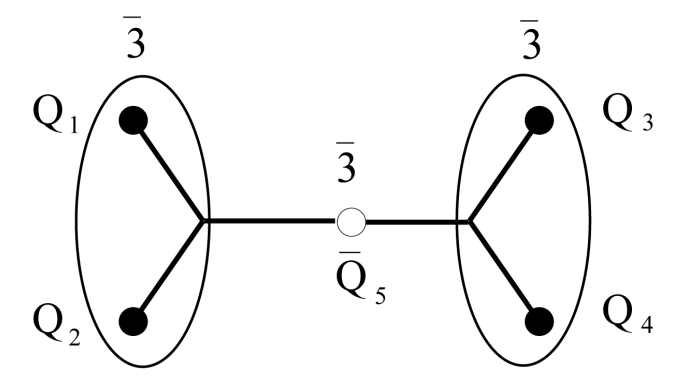

Figure 7. A QQ- $\bar{Q}-Q Q$ type pentaquark configuration [29]. In the $5 \mathrm{Q}$ system, $\left(Q_{1}, Q_{2}\right)$ and $\left(Q_{3}, Q_{4}\right)$ form 3 representation of SU(3) color, respectively. The lattice QCD results indicate the multi-Y-shaped flux-tube formation in the $Q Q-\bar{Q}$ -QQ system.

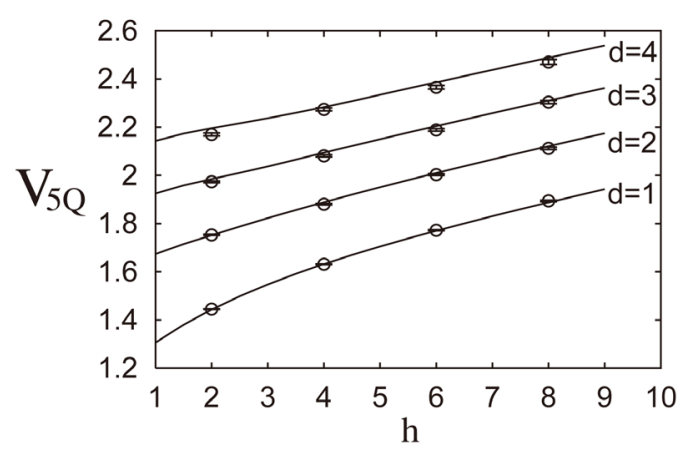

(a)

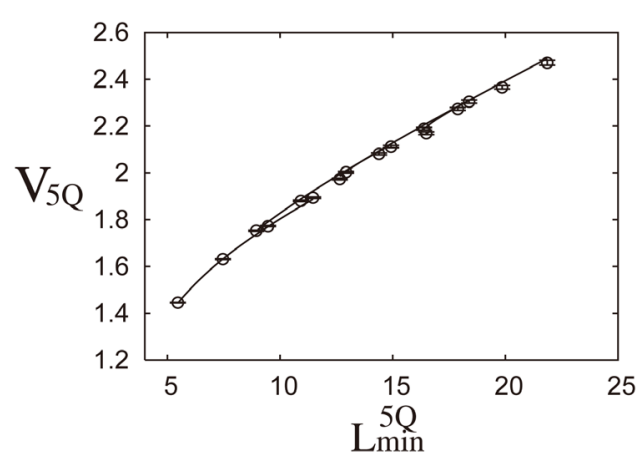

(b)

Figure 8. Lattice QCD results of the pentaquark potential $V_{5 \mathrm{Q}}$ for symmetric planar 5Q configurations in the lattice unit: (a) $V_{5 \mathrm{Q}}$ v.s. $(d, h)$ and (b) $V_{5 \mathrm{Q}}$ v.s. $L_{\min }^{5 \mathrm{Q}}$, taken from Ref. [29]. The symbols denote the lattice QCD data, and the curves the theoretical form of the OGE plus multi-Y Ansatz. 


$$
V_{5 \mathrm{Q}}=-A_{5 \mathrm{Q}}\left\{\left(\frac{1}{r_{12}}+\frac{1}{r_{34}}\right)+\frac{1}{2}\left(\frac{1}{r_{15}}+\frac{1}{r_{25}}+\frac{1}{r_{35}}+\frac{1}{r_{45}}\right)+\frac{1}{4}\left(\frac{1}{r_{13}}+\frac{1}{r_{14}}+\frac{1}{r_{23}}+\frac{1}{r_{24}}\right)\right\}+\sigma_{5 \mathrm{Q}} L_{\min }^{5 \mathrm{Q}}+C_{5 \mathrm{Q}},
$$

where $r_{i j}$ is the distance between $\mathrm{Q}_{i}$ and $\mathrm{Q}_{j}$. Here, $L_{\min }^{5 \mathrm{Q}}$ is the minimal total length of the flux tube, which is multi-Y-shaped as shown in Figure 7. This lattice result supports the flux-tube picture for the 5Q system.

\subsection{Tetraquark Potential and Flip-Flop in Lattice QCD}

We study the tetraquark potential $V_{4 \mathrm{Q}}$ in lattice QCD for about 200 different patterns of QQ- $\bar{Q} \overline{\mathrm{Q}}$ configurations, and find the following results [30]-[32].

1. When QQ and $\overline{\mathrm{Q}} \overline{\mathrm{Q}}$ are well separated, the $4 \mathrm{Q}$ potential $V_{4 \mathrm{Q}}$ is well described by the OGE Coulomb plus multi-Y Ansatz, which indicates the multi-Y-shaped flux-tube formation as shown in Figure 9(a).

2. When the nearest quark and antiquark pair is spatially close, the $4 \mathrm{Q}$ potential $V_{4 \mathrm{Q}}$ is well described by the sum of two Q $\bar{Q}$ potentials, which indicates a "two-meson" state as shown in Figure 9(b).

We show in Figure 10 the lattice QCD results of the 4Q potential $V_{4 Q}$ [30] for symmetric planar 4Q configurations as shown in Figure 9, where each $4 \mathrm{Q}$ system is labeled by $d \equiv \overline{\mathrm{Q}_{1} \mathrm{Q}_{2}} / 2$ and $h \equiv \overline{\mathrm{Q}_{1} \mathrm{Q}_{3}}$.

For large value of $h$ compared with $d$, the lattice data seem to coincide with the solid curve of the OGE Coulomb plus multi-Y Ansatz,

$$
V_{4 \mathrm{Q}}=-A_{4 \mathrm{Q}}\left\{\left(\frac{1}{r_{12}}+\frac{1}{r_{34}}\right)+\frac{1}{2}\left(\frac{1}{r_{13}}+\frac{1}{r_{14}}+\frac{1}{r_{23}}+\frac{1}{r_{24}}\right)\right\}+\sigma_{4 \mathrm{Q}} L_{\min }^{4 \mathrm{Q}}+C_{4 \mathrm{Q}}
$$

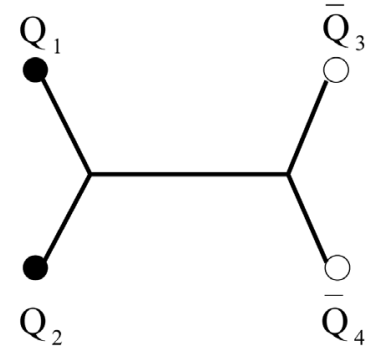

(a)

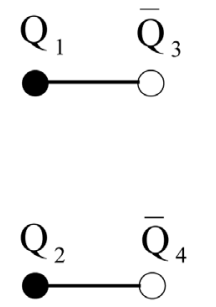

(b)

Figure 9. (a) A connected tetraquark (QQ- $\bar{Q} \bar{Q}$ ) configuration and (b) A “two-meson” configuration [30]. The lattice QCD results indicate the multi-Y-shaped flux-tube formation for the connected $4 \mathrm{Q}$ system.

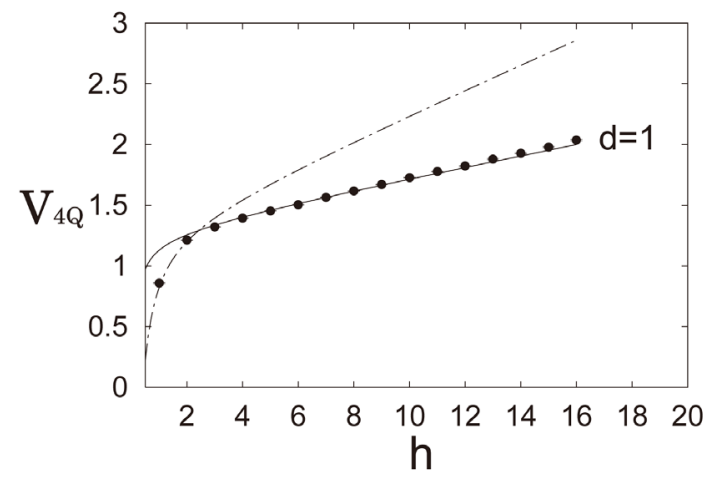

(a)

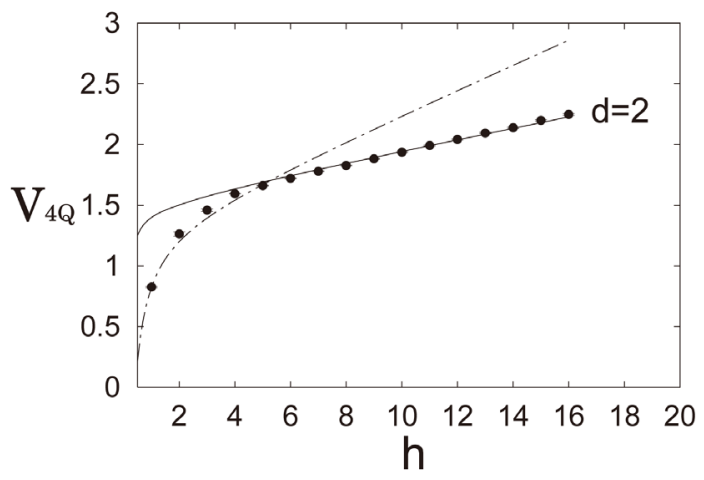

(b)

Figure 10. Lattice QCD results of the tetraquark potential $V_{4 \mathrm{Q}}$ for symmetric planar $4 \mathrm{Q}$ configurations in the lattice unit, taken from Ref. [30]. The symbols denote the lattice QCD data. The solid curve denotes the OGE plus multi-Y Ansatz, and the dotted-dashed curve the two-meson Ansatz. 
where $L_{\min }^{4 Q}$ is the minimal total length of the flux tube, which is multi-Y-shaped as shown in Figure 9(a). Here, the coefficients $\left(A_{4 \mathrm{Q}}, \sigma_{4 \mathrm{Q}}\right)$ are set to be $\left(A_{3 \mathrm{Q}}, \sigma_{3 \mathrm{Q}}\right)$ obtained from the 3Q potential [26].

For small $h$, the lattice data tend to agree with the dotted-dashed curve of the "two-meson" Ansatz, where the $4 \mathrm{Q}$ potential is described by the sum of two $\mathrm{Q} \overline{\mathrm{Q}}$ potentials as $V_{\mathrm{Q} \overline{\mathrm{Q}}}\left(r_{13}\right)+V_{\mathrm{Q} \overline{\mathrm{Q}}}\left(r_{24}\right)=2 V_{\mathrm{Q} \overline{\mathrm{Q}}}(h)$.

Thus, the tetraquark potential $V_{4 Q}$ is found to take the smaller energy of the connected $4 \mathrm{Q}$ state or the two-meson state. In other words, we observe a clear lattice QCD evidence of the "flip-flop", i.e., the flux-tube recombination between the connected $4 \mathrm{Q}$ state and the two-meson state. This lattice result also supports the flux-tube picture for the $4 \mathrm{Q}$ system.

\subsection{Proper Quark-Model Hamiltonian for Multi-Quarks}

From a series of our lattice QCD studies [25]-[33] on the inter-quark potentials, the inter-quark potential is clarified to consist of the one-gluon-exchange (OGE) Coulomb part and the flux-tube-type linear confinement part in $\mathrm{Q} \overline{\mathrm{Q}}$-mesons, 3Q-baryons and multi-quark (4Q, 5Q) hadrons.

Furthermore, from the comparison among the $\mathrm{Q} \overline{\mathrm{Q}}, 3 \mathrm{Q}, 4 \mathrm{Q}$ and $5 \mathrm{Q}$ potentials in lattice $\mathrm{QCD}$, we find the universality of the string tension $\sigma$,

$$
\sigma_{\mathrm{QQ}} \simeq \sigma_{3 \mathrm{Q}} \simeq \sigma_{4 \mathrm{Q}} \simeq \sigma_{5 \mathrm{Q}}
$$

and the OGE result of the Coulomb coefficient $A$ as

$$
\frac{1}{2} A_{\mathrm{QQ}} \simeq A_{3 \mathrm{Q}} \simeq A_{4 \mathrm{Q}} \simeq A_{5 \mathrm{Q}}
$$

in Equations (5), (6), (12) and (13).

Here, the OGE Coulomb term is considered to originate from the OGE process, which plays the dominant role at short distances, where perturbative QCD is applicable. The flux-tube-type linear confinement would be physically interpreted by the flux-tube picture, where quarks and antiquarks are linked by the one-dimensional squeezed color-electric flux tube with the string tension $\sigma$.

To conclude, the inter-quark interaction would be generally described by the sum of the short-distance two-body OGE part and the long-distance flux-tube-type linear confinement part with the universal string tension $\sigma \simeq 0.89 \mathrm{GeV} / \mathrm{fm}$.

Thus, based on the lattice QCD results, we propose the proper quark-model Hamiltonian $\hat{H}$ for multi-quark hadrons as

$$
\hat{H}=\sum_{i} \sqrt{\hat{\mathbf{p}}_{i}^{2}+M_{i}^{2}}+\sum_{i<j} V_{\mathrm{OGE}}^{i j}+\sigma L_{\min },
$$

where $L_{\min }$ is the minimal total length of the flux tube linking quarks. $V_{\text {OGE }}^{i j}$ denotes the OGE potential between $i$ th and $j$ th quarks, which becomes the OGE Coulomb potential in Equation (7) in the static case. $M_{i}$ denotes the constituent quark mass. The semi-relativistic treatment would be necessary for light quark systems.

It is desired to investigate various properties of multi-quark hadrons with this QCD-based quark model Hamiltonian $\hat{H}$.

\section{Summary and Concluding Remarks}

We have studied tetraquark and pentaquark systems in lattice QCD Monte Carlo simulations, motivated by the experimental discoveries of multi-quark candidates.

First, we have performed accurate mass calculations of low-lying 5Q states with $J=1 / 2$ and $I=0$ in both positive- and negative-parity channels in anisotropic lattice QCD. We have found that the lowest positive-parity $5 \mathrm{Q}$ state has a large mass of about $2.24 \mathrm{GeV}$ after the chiral extrapolation. To single out the compact 5Q state from NK scattering states, we have used the hybrid boundary condition (HBC) method, and have found no evidence of the compact $5 \mathrm{Q}$ state below $1.75 \mathrm{GeV}$ in the negative-parity channel.

Second, we have studied the multi-quark potential in lattice QCD to clarify the inter-quark interaction in 
multi-quark systems. We have found that the $5 \mathrm{Q}$ potential $V_{5 \mathrm{Q}}$ for the QQ- $\overline{\mathrm{Q}}-\mathrm{QQ}$ system is well described by the "OGE Coulomb plus multi-Y Ansatz": the sum of the one-gluon-exchange (OGE) Coulomb term and the multi-Y-type linear term based on the flux-tube picture. The $4 \mathrm{Q}$ potential $V_{4 \mathrm{Q}}$ for the QQ- $\overline{\mathrm{Q}} \overline{\mathrm{Q}}$ system is also described by the OGE Coulomb plus multi-Y Ansatz, when QQ and $\bar{Q} \bar{Q}$ are well separated. On the other hand, the $4 \mathrm{Q}$ system is described as a "two-meson" state with disconnected flux tubes, when the nearest quark and antiquark pair is spatially close. We have observed a lattice-QCD evidence for the "flip-flop", i.e., the flux-tube recombination between the connected $4 \mathrm{Q}$ state and the "two-meson" state. On the confinement mechanism, we have clarified the flux-tube-type linear confinement in multi-quark hadrons. Finally, we have proposed the proper quark-model Hamiltonian based on the lattice QCD results.

\section{Acknowledgements}

This paper is based on the unpublished proceeding (hep-ph/0507187, talk by F.O.) at International Workshop on Quark Nuclear Physics, 22-24 Feb 2005. Phoenix Park, Korea. The lattice QCD Monte Carlo calculations were performed on supercomputers at Osaka University and at KEK.

\section{References}

[1] Nakano, T., et al., LEPS Collaboration (2003) Physical Review Letters, 91, Article ID: 012002. http://dx.doi.org/10.1103/PhysRevLett.91.012002

[2] Barmin, V.V., et al., DIANA Collaboration (2003) Physics of Atomic Nuclei, 66, 1715. http://dx.doi.org/10.1134/1.1611587

[3] Stephanyan, S., et al., CLAS Collaboration (2003) Physical Review Letters, 91, Article ID: 252001. http://dx.doi.org/10.1103/PhysRevLett.91.252001

[4] Barth, J., et al., SAPHIR Collaboration (2003) Physics Letters, B572, 127. http://dx.doi.org/10.1016/j.physletb.2003.08.019

[5] Alt, C., et al., NA49 Collaboration (2004) Physical Review Letters, 92, Article ID: 042003. http://dx.doi.org/10.1103/PhysRevLett.92.042003

[6] Aktas, A., et al., H1 Collaboration (2004) Physics Letters, B588, 17. http://dx.doi.org/10.1016/j.physletb.2004.03.012

[7] Schael, S., et al., ALEPH Collaboration (2004) Physics Letters, B599, 1. http://dx.doi.org/10.1016/j.physletb.2004.08.021

[8] Bai, J.X., et al., BES Collabolation (2004) Physical Review, D70, Article ID: 012004. http://dx.doi.org/10.1103/PhysRevD.70.012004

[9] Abt, I., et al., HERA-B Collaboration (2004) Physical Review Letters, 93, Article ID: 212003. http://dx.doi.org/10.1103/PhysRevLett.93.212003

[10] Naruki, M. (2009) Lecture Notes in Physics, 781, 139, and Its References. http://dx.doi.org/10.1007/978-3-642-00961-7_6

[11] Moritsu, M., et al., J-PARC E19 Collaboration (2014) Physical Review C, 90, Article ID: 035205. http://dx.doi.org/10.1103/PhysRevC.90.035205

[12] Choi, S.K., et al., Belle Collaboration (2003) Physical Review Letters, 91, Article ID: 262001. http://dx.doi.org/10.1103/PhysRevLett.91.262001

[13] Acosta, D., et al., CDF II Collaboration (2004) Physical Review Letters, 93, Article ID: 072001. http://dx.doi.org/10.1103/PhysRevLett.93.072001

[14] Abazov, V.M., et al., D0 Collaboration (2004) Physical Review Letters, 93, Article ID: 162002. http://dx.doi.org/10.1103/PhysRevLett.93.162002

[15] Aubert, B., et al., BABAR Collaboration (2004) Physical Review Letters, 93, Article ID: 041801. http://dx.doi.org/10.1103/PhysRevLett.93.041801

[16] Aubert, B., et al., BABAR Collaboration (2003) Physical Review Letters, 90, Article ID: 242001. http://dx.doi.org/10.1103/PhysRevLett.90.242001

[17] Krokovny, P., et al., Belle Collaboration (2003) Physical Review Letters, 91, Article ID: 262002. http://dx.doi.org/10.1103/PhysRevLett.91.262002

[18] Aaij, R., et al., LHCb Collaboration (2015) Physical Review Letters, 115, Article ID: 072001. 
http://dx.doi.org/10.1103/PhysRevLett.115.072001

[19] Diakonov, D., Petrov, V. and Polyakov, M.V. (1997) Zeitschrift für Physik A Hadrons and Nuclei, 359, 305-314. http://dx.doi.org/10.1103/PhysRevLett.115.072001

[20] Jaffe, R.L. and Wilczek, F. (2003) Physical Review Letters, 91, Article ID: 232003. http://dx.doi.org/10.1103/PhysRevLett.91.232003

[21] Stancu, F. and Riska, D.O. (2003) Physics Letters B, 575, 242-248. http://dx.doi.org/10.1016/j.physletb.2003.09.061

[22] Sugiyama, J., Doi, T. and Oka, M. (2004) Physics Letters B, 581, 167-174. http://dx.doi.org/10.1016/j.physletb.2003.12.018

[23] Creutz, M. (1979) Physical Review Letters, 43, 553-556. http://dx.doi.org/10.1103/PhysRevLett.43.553 Creutz, M. (1980) Physical Review D, 21, 2308-2315. http://dx.doi.org/10.1103/PhysRevD.21.2308

[24] Rothe, H.J. (2012) Lattice Gauge Theories. 4th Edition, World Scientific, Singapore. http://dx.doi.org/10.1142/8229

[25] Takahashi, T.T., Matsufuru, H., Nemoto, Y. and Suganuma, H. (2001) Physical Review Letters, 86, 18-21. http://dx.doi.org/10.1103/PhysRevLett.86.18

[26] Takahashi, T.T., Suganuma, H., Nemoto, Y. and Matsufuru, H. (2002) Physical Review D, 65, Article ID: 114509. http://dx.doi.org/10.1103/PhysRevD.65.114509

[27] Takahashi, T.T. and Suganuma, H. (2003) Physical Review Letters, 90, Article ID: 182001. http://dx.doi.org/10.1103/PhysRevLett.90.182001 Takahashi, T.T. and Suganuma, H. (2004) Physical Review D, 70, Article ID: 074506. http://dx.doi.org/10.1103/PhysRevD.70.074506

[28] Sakumichi, N. and Suganuma, H. (2015) Physical Review D, 92, Article ID: 034511. http://dx.doi.org/10.1103/PhysRevD.92.034511

[29] Okiharu, F., Suganuma, H. and Takahashi, T.T. (2005) Physical Review Letters, 94, Article ID: 192001. http://dx.doi.org/10.1103/PhysRevLett.94.192001

[30] Okiharu, F., Suganuma, H. and Takahashi, T.T. (2005) Physical Review D, 72, Article ID: 014505. http://dx.doi.org/10.1103/PhysRevD.72.014505

[31] Suganuma, H., Takahashi, T.T., Okiharu, F. and Ichie, H. (2005) AIP Conference Proceedings, 756, 123. http://dx.doi.org/10.1063/1.1920939

Suganuma, H., Takahashi, T.T., Okiharu, F. and Ichie, H. (2005) Nuclear Physics B_-Proceedings Supplements, 141, 92-98. http://dx.doi.org/10.1016/j.nuclphysbps.2004.12.015

[32] Okiharu, F., Suganuma, H. and Takahashi, T.T. (2005) The Static Pentaquark Potential in Lattice QCD. Proceedings of the International Workshop on Pentaquark 04, SPring-8, Hyogo, 20-23 July 2004, 339-343.

[33] Suganuma, H., Okiharu, F., Takahashi, T.T. and Ichie, H. (2005) Nuclear Physics A, 755, 399-402. http://dx.doi.org/10.1016/j.nuclphysa.2005.03.098

[34] Ishii, N., Doi, T., Iida, H., Oka, M., Okiharu, F. and Suganuma, H. (2005) Physical Review D, 71, Article ID: 034001. http://dx.doi.org/10.1103/PhysRevD.71.034001

[35] Ishii, N., Doi, T., Iida, H., Oka, M., Okiharu, F. and Suganuma, H. (2005) Nuclear Physics A, 755, 467-470. http://dx.doi.org/10.1016/j.nuclphysa.2005.03.057

[36] Zhu, S.L. (2004) International Journal of Modern Physics A, 19, 3439-3469. http://dx.doi.org/10.1142/S0217751X04019676

[37] Oka, M. (2004) Progress of Theoretical Physics, 112, 1-19. http://dx.doi.org/10.1143/PTP.112.1

[38] Csikor, F., Fodor, Z., Katz, S.D. and Kovacs, T.G. (2003) Journal of High Energy Physics, 2003, 070.

[39] Sasaki, S. (2004) Physical Review Letters, 93, Article ID: 152001. http://dx.doi.org/10.1103/PhysRevLett.93.152001

[40] Chiu, T.W. and Hsieh, T.H. (2005) Physical Review D, 72, Article ID: 034505. http://dx.doi.org/10.1103/PhysRevD.72.034505

[41] Mathur, N., Lee, F.X., Alexandru, A., Bennhold, C., Chen, Y., Dong, S.J., Draper, T., Horváth, I., Liu, K.F., Tamhankar, S. and Zang, J.B. (2004) Physical Review D, 70, Article ID: 074508. http://dx.doi.org/10.1103/PhysRevD.70.074508

[42] Takahashi, T.T., Umeda, T., Onogi, T. and Kunihiro, T. (2005) Physical Review D, 71, Article ID: 114509. http://dx.doi.org/10.1103/PhysRevD.71.114509

[43] Ishii, N., Doi, T., Nemoto, Y., Oka, M. and Suganuma, H. (2005) Physical Review D, 72, Article ID: 074503. http://dx.doi.org/10.1103/PhysRevD.72.074503

[44] Capstick, S., Page, P.R. and Roberts, W. (2003) Physics Letters B, 570, 185-190. 
http://dx.doi.org/10.1016/j.physletb.2003.08.010

[45] Hosaka, A. (2003) Physics Letters B, 571, 55-60. http://dx.doi.org/10.1016/j.physletb.2003.07.067

[46] Kanada-Enyo, Y., Morimatsu, O. and Nishikawa, T. (2005) Physical Review C, 71, Article ID: 045202. http://dx.doi.org/10.1103/PhysRevC.71.045202

[47] Karliner, M. and Lipkin, H.J. (2003) Physics Letters B, 575, 249-255. http://dx.doi.org/10.1016/j.physletb.2003.09.062

[48] Bicudo, P. and Marques, G.M. (2004) Physical Review D, 69, Article ID: 011503. http://dx.doi.org/10.1103/PhysRevD.69.011503

[49] Sakumichi, N. and Suganuma, H. (2014) Physical Review D, 90, Article ID: 111501(R). http://dx.doi.org/10.1103/PhysRevD.90.111501

[50] Nambu, Y. (1974) Physical Review D, 10, 4262-4268. http://dx.doi.org/10.1103/PhysRevD.10.4262

[51] Kogut, J. and Susskind, L. (1975) Physical Review D, 11, 395-408. http://dx.doi.org/10.1103/PhysRevD.11.395

[52] Casher, A., Neuberger, H. and Nussinov, S. (1979) Physical Review D, 20, 179-188. http://dx.doi.org/10.1103/PhysRevD.20.179

[53] Carlson, J., Kogut, J.B. and Pandharipande, V.R. (1983) Physical Review D, 27, 233-243. http://dx.doi.org/10.1103/PhysRevD.27.233 Carlson, J., Kogut, J.B. and Pandharipande, V.R. (1983) Physical Review D, 28, 2807-2817. http://dx.doi.org/10.1103/PhysRevD.28.2807

[54] Ichie, H., Bornyakov, V., Streuer, T. and Schierholz, G. (2003) Nuclear Physics A, 721, C899-C902. http://dx.doi.org/10.1016/S0375-9474(03)01238-7

[55] Kuzmenko, D.S. and Simonov, Y.A. (2003) Physics of Atomic Nuclei, 66, 950-954. http://dx.doi.org/10.1134/1.1577917

[56] Bowman, P.O. and Szczepaniak, A.P. (2004) Physical Review D, 70, Article ID: 016002. http://dx.doi.org/10.1103/PhysRevD.70.016002

[57] Cornwall, J.M. (2004) Physical Review D, 69, Article ID: 065013. http://dx.doi.org/10.1103/PhysRevD.69.065013 Cornwall, J.M. (2005) Physical Review D, 71, Article ID: 056002. http://dx.doi.org/10.1103/PhysRevD.71.056002 